\title{
Droplets size evolution of dispersion in a stirred tank
}

\author{
Bohus Kysela $^{1 \text { a }}$, Jiri Konfrst ${ }^{1}$, Zdenek Chara ${ }^{1}$, Radek Sulc ${ }^{2}$, and Darina Jasikova ${ }^{3}$ \\ 1 The Czech Academy of Sciences, Institute of Hydrodynamics \\ Pod Patankou 30/5, 16612 Prague 6, Czech Republic \\ 2 Czech Technical University in Prague, Faculty of Mechanical Enginnering, Department of Process Engineering, \\ Technicka 4, 16607 Prague 6, Czech Republic \\ 3 Technical University of Liberec, Institute for Nanomaterials, Advanced Technology and Innovation, \\ Studentska 1402/2, 46117 Liberec, Czech Republic
}

\begin{abstract}
Dispersion of two immiscible liquids is commonly used in chemical industry as wall as in metallurgical industry e. g. extraction process. The governing property is droplet size distribution. The droplet sizes are given by the physical properties of both liquids and flow properties inside a stirred tank. The first investigation stage is focused on in-situ droplet size measurement using image analysis and optimizing of the evaluation method to achieve maximal result reproducibility. The obtained experimental results are compared with multiphase flow simulation based on Euler-Euler approach combined with PBM (Population Balance Modelling). The population balance model was, in that specific case, simplified with assumption of pure breakage of droplets.
\end{abstract}

\section{Introduction}

Particle size distribution is a crucial property in particulate processes and it affects a lot of quantities - flow of suspension, dispersion, eventuallly separation processes, mass transfer etc. This contribution is focused on in-situ measurements and evaluation of particle size distribution of suspended solid spherical particles and spherical droplets of oil in water-oil dispersion. The dispersions were processed in an agitated vessel. These investigated processes are commonly used in chemical or metallurgical industry (e.g. extraction, catalytic reactions, polymerization etc.). The dynamic evolution of droplet size distribution in time inside an agitated vessel has been investigated by many researchers [1-5]. They tried to develop a simple model of droplet size distribution developing in time and depending on parameters of the mixing system. The agitated vessel geometry or impeller types were also examined [5,4]. Several approaches applying of population balance modelling [6-9] were used. The generalized equation of population balance can be used to solve grow of crystals (molecular transport, nucleation, breakage, aggregation), aggregation process of solid particles in fluid, liquid-liquid dispersion process and many others. The method is based on the solution of integro-differential equations with probability density functions. There are many methods how to solve population balance, one of the commonly used is the QMOM (Quadrature method of moments) based on quadrature approximation $[10,11]$ which can be very easily implemented to existing solvers based on finite volume method. Hence it is used in coupling with CFD (Computational fluid dynamics). The coupling is based on media transport, application of turbulence diffusion as well as application of dissipation rate of turbulent kinetic energy in breakage kernel functions. The main aim of our investigation is development of very precise measurement of the

a e-mail: kysela@ih.cas.cz

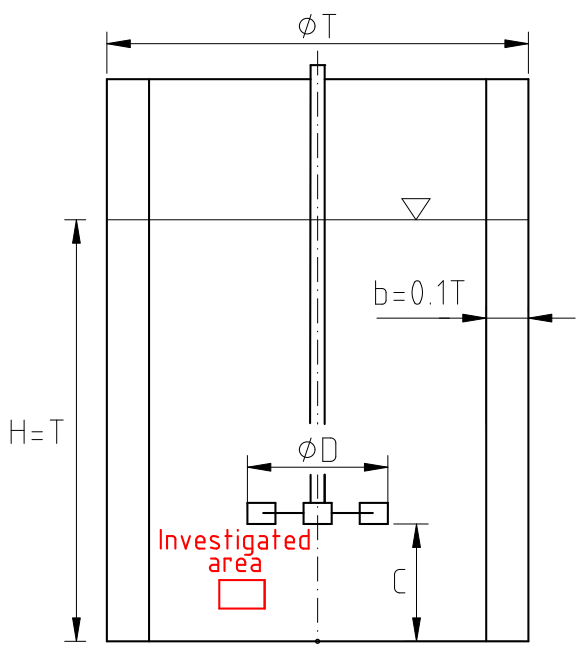

Fig. 1. Geometry of the mixing tank $(T=300 \mathrm{~mm}, \mathrm{H} / \mathrm{T}=1, \mathrm{~T} / \mathrm{D}$ $=3, \mathrm{~b} / \mathrm{T}=1 / 10$, four baffles).

droplet size distribution in a pseudo steady state, when the system is in balance and particle size distribution is stable (balance for one impeller speed in agitated vessel system), and assumed breakage process only. Then the initial conditions and calculation setting could by adjusted to the numerical simulation. The next step is validation of the time dependent evolution droplet size modelling in future.

\section{Droplet size measurement}

Measurement was performed using two immiscible liquids - water and silicone oil in an agitated system schematically described in Fig.1. The particle sizes were evaluated from the raw image records by the image analysis. 


\subsection{Experimental set-up}

The experiments were performed in the agitated vessel with standard cylindrical geometry and with the flat bottom. The vessel was equipped with four radial baffles (width $b=0.1$ $\mathrm{T})$. The diameter of the vessel was $\mathrm{T}=300 \mathrm{~mm}$. The vessel was filled with water and the liquid level was $\mathrm{H}=\mathrm{T}$. The standard Rushton impeller with ratio T/D $=3$ and offbottom clearance $\mathrm{C}=0.75 \mathrm{D}$ (see Fig.1) was used. The impeller speed was $150 ; 200 ; 250 ; 300 \mathrm{rpm}$. The model liquids were distilled water and silicone oil AP 200 (density $=1070 \mathrm{~kg} \cdot \mathrm{m}^{3}$, kinematic viscosity $=2 \cdot 10^{-4} \mathrm{~m}^{2} \cdot \mathrm{s}^{-1}$ at $25^{\circ} \mathrm{C}$ ). Used dispersed oil phase fraction was 0.0047 , where the oil was added in before measurement by syringe injection. The size of the investigated area (Fig.1) was approximately $15 \times 12 \mathrm{~mm}$. The area was located $40 \mathrm{~mm}$ from impeller axis and off-bottom clearance was $30 \mathrm{~mm}$. The focused plane was approximately $50 \mathrm{~mm}$ from the vessel wall. This position was chosen due to the circulation loop generated by the radial impeller. The dispersion in that place is moving uniformly.

\subsection{Image recording}

The image recording was realised by a high speed camera system SpeedSence MK III with frame rate lowered to $30 \mathrm{fps}$, shutter time $0.1 \mathrm{~ms}$, full resolution $1280 \times$ 1024 pixels. The frames were captured at the region under the impeller (see Fig. 1), where the particle velocities were below $1 \mathrm{~m} \cdot \mathrm{s}^{-1}$. The image resolution was $0.0118 \mathrm{~mm} / \mathrm{pixel}$ in this configuration. Each record was 1000 images (total time $33.3 \mathrm{~s}$ ) and time of $10 \mathrm{~min}$. was taken between impeller speed changes to stabilise the droplet distribution.

\subsection{Image analysis}

The detected objects evaluation was investigated by several approaches. At first the illumination of the captured area was tested. The light sheets as well as various light sources were used. The light scattering and refraction trough the transparent droplets disallowed precise evaluation. Moreover, it was also shown that the evaluation by a standard threshold was not quite accurate. The back lighting combined with enhanced approach of image evaluation based on finding edges of spherical particles was found as the best final configuration (see Fig. 2. The three basic conditions had to be fulfilled:

i) Only different particles should be detected on the subsequent frames (that is why frame rate was only 30 fps).

ii) The moving particles should have sharp boundary (very short shutter time is necessary as well as high-power light source)

iii) Using precise spherical particles or providing droplets with minimal deformation.

The final approach for the image analysis was: 1) edges finding (high gradients), 2) threshold, 3) evaluation of particle area using fill-holes filter and enhanced by circularity evaluation.

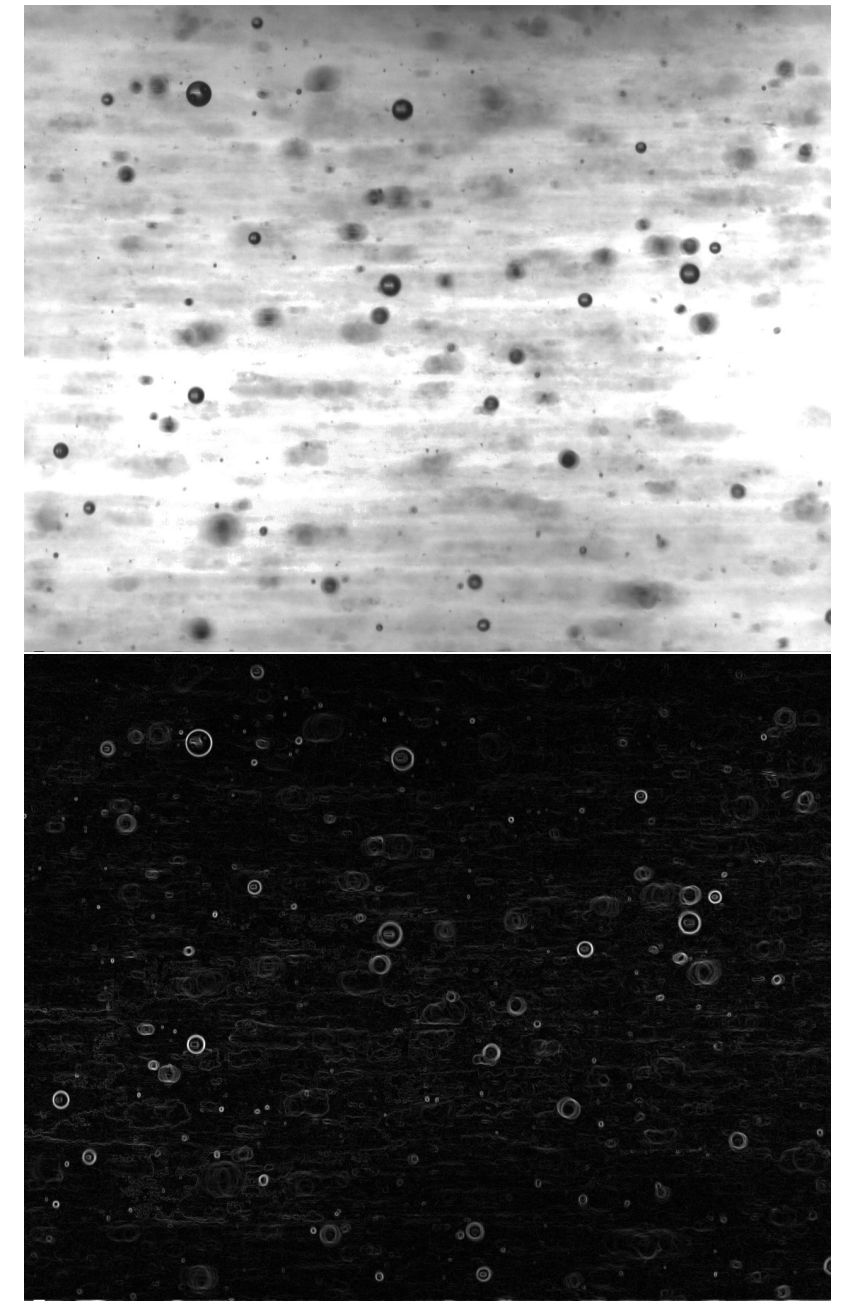

O

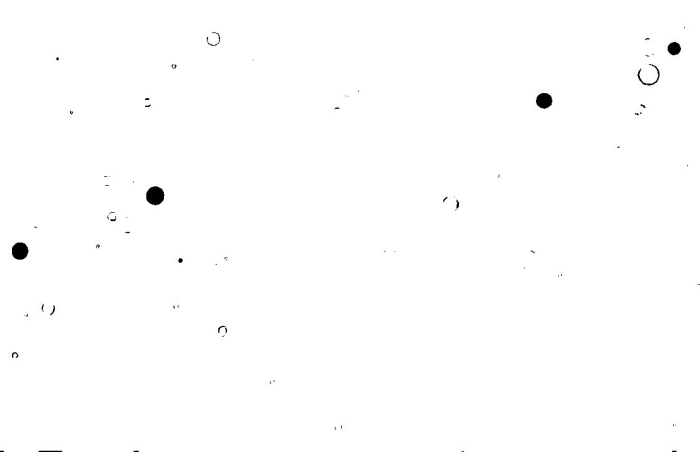

Fig. 2. Example of obtained raw image (top), finding edges - high gradients (middle), and evaluated result (bottom).

\section{Population balance modelling (PBM)}

The dispersion process of the oil droplets was also modelled using CFD-PBM approach. The special add-on of ANSYS Fluent software package was used. The numerical simulations were performed using combination of fluid flow solution in the modelled agitated vessel and implemented PBM solution. 


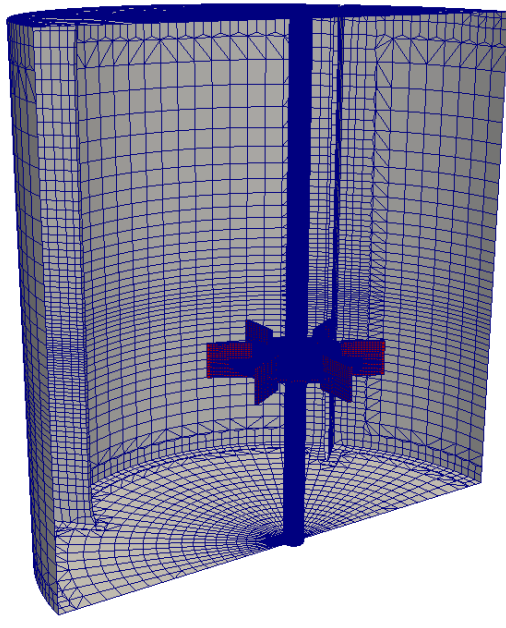

Fig. 3. Mesh of the agitated vessel and Rushton turbine impeller.

The flow was solved with transient RANS model k- $\varepsilon$ Realizable combined with MRF(moving reference frame) method for simulation of the impeller movement. The MRF region has the cylindrical shape with distance $\mathrm{D} / 10$ under and below impeller cylindrical envelope and with diameter 2D. The mesh size was approximately 0.5 mil. mainly tetrahedral cells more dense in impeller region (Fig. 3). Two types of boundary conditions were defined: no slip walls (impeller, tank) and moving wall (impeller shaft outside of MRF zone). Standard wall functions were used for the walls. The population balance is given in that specific case only by the breakage of droplets without nucleation and molecular growth and also without aggregation because the droplets are in very low concentration. Thus the population balance [9] can be simplified:

$$
\begin{aligned}
\frac{\partial n(L ; \mathbf{x}, t)}{\partial t}+\left\langle u_{i}\right\rangle \frac{\partial n(L ; \mathbf{x}, t)}{\partial x_{i}} & -\frac{\partial}{\partial x_{i}}\left[\Gamma_{t} \frac{\partial n(L ; \mathbf{x}, t)}{\partial x_{i}}\right] \\
& =B(L ; \mathbf{x}, t)-D(L ; \mathbf{x}, t),
\end{aligned}
$$

where $n(L ; \mathbf{x}, t)$ is particle size distribution function, and $L$ represents particle size, $\mathbf{x}$ is position vector, $t$ is time. This equation is specified for combination with CFD calculations and $\left\langle u_{i}\right\rangle$ is Reynolds-averaged velocity in the $i$-th direction, and $\Gamma_{t}$ represents turbulent diffusivity. The terms on the left side $B(L ; \mathbf{x}, t)$ and $D(L ; \mathbf{x}, t)$ are, respectively, the bird and death rates due to breakage. The moments of the PSD are defined as follows:

$$
m_{k}(\mathbf{x}, t)=\int_{0}^{\infty} n(L ; \mathbf{x}, t) L^{k} \mathrm{~d} L
$$

and thus the transport equation for the $k$-th moment is

$$
\begin{aligned}
\frac{\partial m_{k}(\mathbf{x}, t)}{\partial t}+\left\langle u_{i}\right\rangle \frac{\partial m_{k}(\mathbf{x}, t)}{\partial x_{i}} & -\frac{\partial}{\partial x_{i}}\left[\Gamma_{t} \frac{\partial m_{k}(\mathbf{x}, t)}{\partial x_{i}}\right] \\
& =\overline{B_{k}}(\mathbf{x}, t)-\overline{D_{k}}(\mathbf{x}, t),
\end{aligned}
$$

where the moments of the bird and death are

$$
\overline{B_{k}}(\mathbf{x}, t)=\int_{0}^{+\infty} L_{k} \int_{0}^{+\infty} a(\lambda) b(L \mid \lambda) n(\lambda ; \mathbf{x}, t) \mathrm{d} \lambda \mathrm{d} L,
$$

$$
\overline{D_{k}}(\mathbf{x}, t)=\int_{0}^{+\infty} L^{k} a(L) n(L ; \mathbf{x}, t) \mathrm{d} L .
$$

The breakage kernel $a(L)$ defines the rate coefficient for breakage particle of the length $L$. The fragment distribution function for the breakage of a particle of size $L$ is given by $b(L \mid \lambda)$, where $L$ and $\lambda$ are lengths of two particles. The breakage kernel was used Luo-Svensen [12] for liquid-liquid system:

$a(L)=c_{4}\left(\frac{\varepsilon}{L^{2}}\right)^{1 / 3} \int_{\xi_{\min }}^{1} \frac{(1+\xi)^{2}}{\xi^{11 / 3}} \exp \left[-\frac{12 c_{f} \sigma}{\beta \rho_{d} \varepsilon^{2 / 3} L^{5 / 3} \xi^{11 / 3}}\right] \mathrm{d} \xi$

where the $\xi=\eta / L, \eta$ is the eddy length-scale, $c_{4}$ is a constant of order unity $\left(c_{4}=0.92\right), c_{f}$ is the increase coefficient in the surface area $\left[c_{f}=f^{2 / 3}+(1-f)^{2 / 3}-1\right]$ and $f$ is the volume fraction of one of two fragments.

The quadrature method of moments (QMOM) is based on the following quadrature approximation:

$$
m_{k}(\mathbf{x}, t)=\int_{0}^{\infty} n(L) L^{k} \mathrm{~d} L \approx \sum_{i=1}^{N_{d}} w_{i} L_{i}^{k}
$$

where weights $\left(w_{i}\right)$ and abscissas $\left(L_{i}\right)$ are determined through the product-difference (PD) algorithm from the low-order moments [9]. By using the PD algorithm, a quadrature approximation with $N_{d}$ weights and $N_{d}$ abscissas can be constructed using the first $2 N_{d}$ moments of PSD. For example, if $N_{d}=3$ only the first six moments $\left(m_{0}, \ldots ., m_{5}\right)$ are tracked, and the quadrature approximation is given by

$$
m_{k}=\sum_{i=1}^{N_{d}=3} w_{i} L_{i}^{k}=w_{1} L_{1}^{k}+w_{2} L_{2}^{k}+w_{3} L_{3}^{k} .
$$

Knowledge of $w_{i}$ and $L_{i}$ suffices to close the transport equations for the moments, and in our case with the presumption of pure breakage the moment equation become

$$
\begin{aligned}
\frac{\partial m_{k}(\mathbf{x}, t)}{\partial t}+\left\langle u_{i}\right\rangle \frac{\partial m_{k}(\mathbf{x}, t)}{\partial x_{i}} & -\frac{\partial}{\partial x_{i}}\left[\Gamma_{t} \frac{\partial m_{k}(\mathbf{x}, t)}{\partial x_{i}}\right] \\
& =\sum_{i} a_{i} \bar{b}_{i}^{(k)} w_{i}-\sum_{i} L_{i}^{k} a_{i} w_{i}
\end{aligned}
$$

where $a_{i}=a\left(L_{i}\right)$, and

$$
\bar{b}_{i}^{(k)}=\int_{0}^{+\infty} L^{k} b\left(L \mid L_{i}\right) \mathrm{d} L .
$$

It has been shown $[9,10]$ that by using this quadrature approximation it is possible to track the moments of the PSD with very high accuracy. For the reconstruction of distribution from the finite number of its moments can be used several techniques $[13,14]$.

\section{Results}

The measurement based on image analysis is limited by a number of evaluated particles. Moreover, the proposed more accurate method this number even decreases. The 


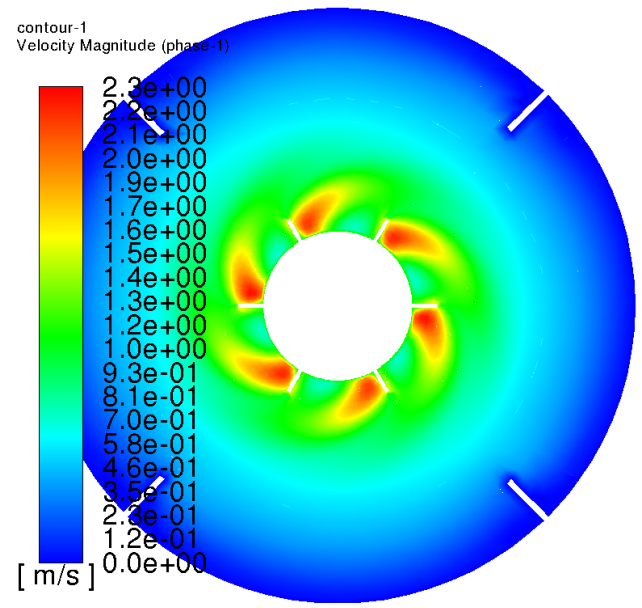

Fig. 4. Distribution of the velocity magnitude in the impeller plane (300rpm).

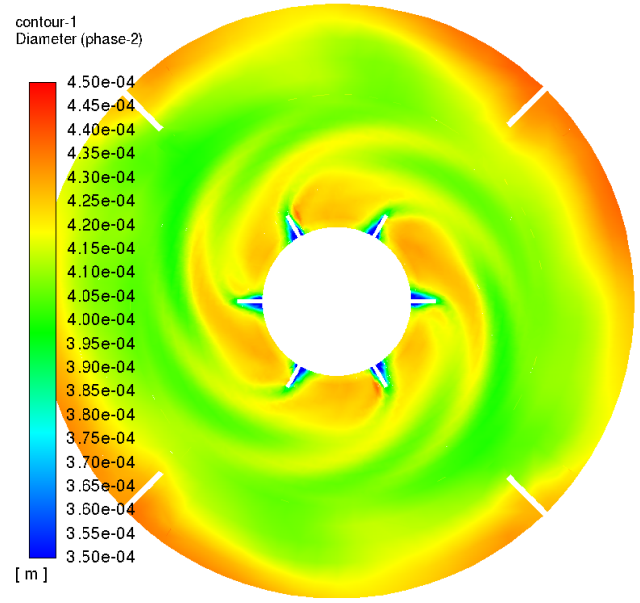

Fig. 5. Distribution of the particle mean Sauter $\left(d_{32}\right)$ diameter in the impeller plane (300rpm).

dependency of mean Sauter droplet diameter on number of droplets was evaluated. The results corresponding to all impeller speeds are depicted in Fig. 6. There are visible changes approximately up to 1000 of evaluated droplets in the case of all impeller speeds. The decrease of mean diameter with number of evaluated droplets at $150 \mathrm{rpm}$ was evidently caused by not absolutely stable state at the beginning of the measurement. The big jump in this dependency was induced by random appearing of several big droplets. It confirms an opinion of unstable beginning state. The real accuracy of image analysis evaluation was investigated using the precise spherical stainless steel particles with diameter $0.635 \pm 0.001 \mathrm{~mm}$. The evaluation process was set in accordance with the best obtained results for the spherical particles. The variance was under $3 \%$. The evolution of droplet volume cumulative distribution with impeller speed is shown in Fig. 7. Depicted results indicate a similar tendency at the increasing impeller speed as transient drop size distribution change obtained by Bak and Podgorska [2] for droplets in liquid-liquid system in an agitated vessel. The real experimental system of agitated ves-

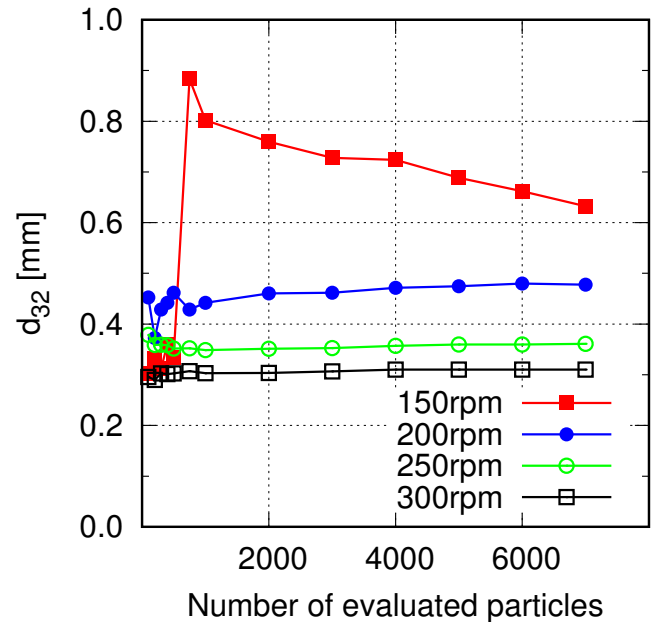

Fig. 6. Mean Sauter $\left(d_{32}\right)$ droplet diameter changes with the number of evaluated particles.

sel was subsequently simulated using CFD-PBM approach with identical geometry and identical conditions (impeller speeds, densities, viscosities etc.). The inter-facial tension between oil and water was determined $\sigma=0.0035 \mathrm{~N} \cdot \mathrm{m}^{-1}$ [15] as an input value for the calculations. The breakage kernel was used Luo-Svensen [12] described in eq. 6. Input distribution of droplets was mono-disperse with $6 \mathrm{~mm}$ in diameter (similar to the syringe dosage in real case). The simulation time was $10 \mathrm{~min}$ as well as in experiments. An example of both results flow field and mean Sauter $\left(d_{32}=m_{3} / m_{2}\right)$ droplets diameter are depicted in Fig. 4 and Fig. 5, respectively. The results are shown in impeller plane, where regions with the maximal shear rates and high turbulent kinetic dissipation rates are usually found. The comparison of the droplet distribution from experiments and calculations are shown in Fig. 7. The distribution at $300 \mathrm{rpm}$ is slightly underestimated by calculations. It could be caused by calculations of the turbulent kinetic energy dissipation rate (that are usually underestimated too) and also by the discrepancy in initial conditions and PBM inputs. The results at the $150 \mathrm{rpm}$ indicate the discrepancy of distribution shape that is probably caused by the nonstable conditions in experimental measurements. Nevertheless, the trend of results for both measurements and simulations is consistent.

\section{Conclusions}

The method of in-situ measurement of particle size distribution based on image analysis was improved with finding edges (high gradients in images) evaluation enhanced by circularity analysis. The high accuracy and good reproducibility allows to apply this method to precise measurement of the drop size distribution. The results were compared with calculations based on the combination of the CFD with the PBM. The results verified that only the breakage process is involved in our case (in very diluted dispersions). But it was found that the final results of CFDPBM calculations are not only dependent on the model settings, but also on correctly defined conditions in comparison with experiments. 


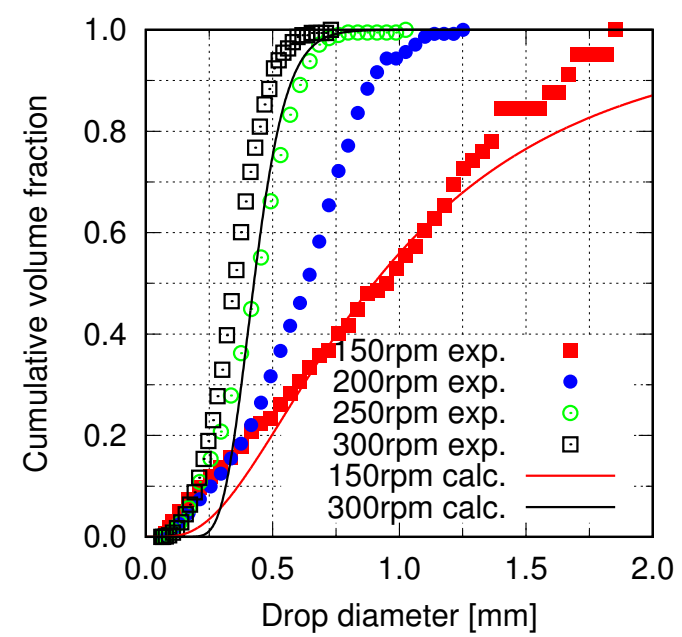

Fig. 7. Relation of the cumulative volume fraction on drop diameter at 150; 200; 250; $300 \mathrm{rpm}$ (points - experiments, continuous line - simulations)

\section{Acknowledgments}

This research has been subsidized by the research project No. GA ČR 16-20175S and RVO: 67985874.

\section{References}

1. J. Baldyga, J. Bourne, A. Pacek, A. Amanullah, A. Nienow, Chemical Engineering Science 56, 3377 (2001)

2. A. Bak, W. Podgarska, Chemical Engineering Research and Design 91, 2142 (2013), mixing

3. P. Ditl, R. Sulc, Technical Transactions. M-1, 83 (2016)

4. G. Zhou, S.M. Kresta, Chemical Engineering Science 53, 2099 (1998)

5. H. Tang, L. Wrobel, International Journal of Engineering Science 43, 1234 (2005)

6. N.B. Raikar, S.R. Bhatia, M.F. Malone, D.J. McClements, C. Almeida-Rivera, P. Bongers, M.A. Henson, Colloids and Surfaces A: Physicochemical and Engineering Aspects 361, 96 (2010)

7. J. Baldyga, W. Orciuch, Chemical Engineering Science 56, 2435 (2001), industrial Crystallisation

8. A. Sathyagal, D. Ramkrishna, G. Narsimhan, Computers and Chemical Engineering 19, 437 (1995)

9. D.L. Marchisio, R.D. Vigil, R.O. Fox, Chemical Engineering Science 58, 3337 (2003)

10. D.L. Marchisio, R. Vigil, R.O. Fox, Journal of Colloid and Interface Science 258, 322 (2003)

11. T. Nguyen, F. Laurent, R. Fox, M. Massot, Journal of Computational Physics 325, 129 (2016)

12. H. Luo, H.F. Svendsen, AIChE Journal 42, 1225 (1996)

13. V. John, I. Angelov, A. Oncul, D. Thevenin, Chemical Engineering Science 62, 2890 (2007)

14. L. de Souza, G. Janiga, V. John, D. Thevenin, Chemical Engineering Science 65, 2741 (2010)

15. F. Peters, D. Arabali, Colloids and Surfaces A: Physicochemical and Engineering Aspects 426, 1 (2013) 